\title{
Dopamine-controlled delta oscillations regulate dynamical and computational regimes in cortical networks Daniel Durstewitz
}

\author{
Address: Central Institute of Mental Health, RG Computational Neuroscience, University of Heidelberg, 68159 Mannheim, Germany \\ Email: Daniel Durstewitz - daniel.durstewitz@zi-mannheim.de
}

from Eighteenth Annual Computational Neuroscience Meeting: CNS*2009

Berlin, Germany. 18-23 July 2009

Published: 13 July 2009

BMC Neuroscience 2009, I0(Suppl I):P269 doi:I0.I I86/I47 I-2202-I0-SI-P269

This abstract is available from: http://www.biomedcentral.com/I47I-2202/I0/SI/P269

(C) 2009 Durstewitz; licensee BioMed Central Ltd.

Delta band $(0.5-3 \mathrm{~Hz})$ oscillations are a prominent phenomenon in cortical dynamics, particularly during slowwave sleep, but even in awake behaving animals [1]. Their functional role, however, is not well understood. Here it is shown through biophysically realistic (compartmental model) network simulations that the neuromodulator dopamine may regulate the dynamical and computational regime of cortical networks via modulation of delta frequency oscillations. Dopamine in prefrontal cortex (PFC) modulates a variety of voltage-gated and synaptic ion channels via D1- and D2-class receptors, and through these cellular and synaptic actions profoundly affects the dynamics of PFC networks [2]. In particular, as shown here, it can lead to the emergence of delta oscillations in the PFC target area through a Hopf bifurcation, and controls their amplitude depending on the ratio of D1/D2 receptor activation. This regulation has a number of implications. In one regime, due to prevalent stimulation of D1-class receptors, network activity is characterized by large-amplitude oscillations and a high degree of order and coherence, while in a D2 receptor-dominated regime network activity is only weakly oscillatory, highly divergent, irregular, and accompanied by a large diversity of spatio-temporal patterns. These different dynamical regimes may in turn bear particular computational advantages. The D1-regime proved to be intrinsically, without explicit embedding of attractor states, more apt for online maintenance of information, while the D2-regime was advantageous for disentangling complex stimulus classes. Hence it is suggested that dopamine may regulate the trade-off between different computational requirements by controlling the $\mathrm{D} 1 / \mathrm{D} 2$ receptor activation ratio, in line with the differential involvement of D1- and D2-type receptors in working memory and cognitive flexibility demonstrated experimentally. Thus the present study may provide new insights into the functional and dynamical role of dopamine in prefrontal cognition, and in addition suggests a novel computational role for delta oscillations in cortical dynamics. If confirmed experimentally, this potentially suggests new routes for the treatment of schizophrenia by manipulating delta oscillations.

\section{Acknowledgements}

This work was supported by grants from the Deutsche Forschungsgemeinschaft (Du 354/5-I \& Du 354/6-I).

\section{References}

I. Spencer KM, Polich J: Poststimulus EEG spectral analysis and P300: attention, task, and probability. Psychophysiology 1999, 36:220-232

2. Durstewitz D, Seamans JK: The computational role of dopamine DI receptors in working memory. Neural Networks 2002, I5:561-572. 